Mots. Les langages du politique

$87 \mid 2008$

Chrononymes. La politisation du temps

Les journalistes politiques et leurs sources. D'une rhétorique de l'expertise critique à une rhétorique du « cynisme»

\title{
Eugénie Saitta
}

\section{CpenEdition}

Journals

Édition électronique

URL : https://journals.openedition.org/mots/12722

DOI : $10.4000 /$ mots. 12722

ISSN : 1960-6001

Éditeur

ENS Éditions

\section{Édition imprimée}

Date de publication : 21 juillet 2008

Pagination : 113-128

ISBN : 978-2-271-06685-5

ISSN : 0243-6450

Référence électronique

Eugénie Saitta, «Les journalistes politiques et leurs sources. D'une rhétorique de l'expertise critique à une rhétorique du « cynisme » », Mots. Les langages du politique [En ligne], 87 | 2008, mis en ligne le 21 juillet 2010, consulté le 22 avril 2022. URL : http://journals.openedition.org/mots/12722 ; DOl : https:// doi.org/10.4000/mots. 12722 


\section{Eugénie Saitta}

\section{Les journalistes politiques et leurs sources. D'une rhétorique de l'expertise critique à une rhétorique du «cynisme »}

Les journalistes politiques français sont - et ce, de façon particulièrement exacerbée par rapport à d'autres spécialités journalistiques - soupçonnés de connivence avec leurs sources ${ }^{1}$. Ils se trouvent en effet confrontés à un climat de défiance au sein des rédactions de la presse quotidienne nationale. Des livres écrits par des journalistes dits d'investigation², mais aussi d'anciens journalistes politiques 3 ou encore des dossiers spéciaux de revue 4 leur reprochent ainsi leur proximité avec le personnel politique et leur traitement déférent et révérencieux de la politique. Ces critiques émises par les pairs se voient confortées par des sondages d'opinion, à l'instar du baromètre annuel Sofres-Télérama-La Croix sur la confiance des Français dans les médias, publié depuis 1988, où les personnes interrogées expriment leur suspicion à l'égard des journalistes et de leur connivence supposée avec les milieux du pouvoir5. En effet, l'autonomie, l'indépendance ou encore l'objectivité deviennent des critères dominants dans la définition de l'excellence journalistique à partir des années quatre-vingt/quatre-vingt-dix.

Ici, il s'agit de comprendre dans quelle mesure la distanciation à l'égard du personnel politique dont se revendiquent les journalistes politiques transforme leurs pratiques de travail quotidiennes et, plus précisément, leurs pratiques d'écriture. Nous avons ainsi constaté l'appropriation - généralisée à l'ensemble des quotidiens nationaux dans les années quatre-vingt-dix de formes textuelles que, traditionnellement, on ne rencontrait pas, ou peu,

Université de Poitiers, CRAPE, eugeniesaitta@yahoo.fr

1. Cet article est issu d'une thèse de science politique soutenue en 2006 à l'IEP de Rennes (Les transformations du journalisme politique depuis les années quatre-vingt. Une comparaison France/Italie).

2. Entre autres, Serge Halimi, 1997, Les nouveaux chiens de garde, Paris, Liber-Raisons d'Agir; Edwy Plenel, 1994, Un temps de chien, Paris, Stock; Denis Robert, 1996, Pendant les " affaires », les affaires continuent..., Paris, Stock.

3. Par exemple, Daniel Carton, 2003, Bien entendu... c'est off, Paris, Albin Michel.

4. Voir par exemple Manière de voir, "Médias et contrôle des esprits », 1995, n² 27 et, en particulier, Serge Halimi, «Un journalisme de révérence», p. 14-17.

5. Voir par exemple Télérama, 27 janvier-2 février 2001. 
dans les formats d'expression préférentiels du journalisme politique. À partir d'une trentaine d'entretiens réalisés avec des journalistes politiques de la presse quotidienne nationale ${ }^{6}$ et d'observations directes au service politique du Monde7, nous avons relevé les formes textuelles identifiées par les journalistes politiques comme les plus gratifiantes et souvent présentées comme l'illustration de leur prise de distance par rapport aux sources.

L'expression d'une distanciation à l'égard des acteurs politiques dans l'écriture de l'information politique n'est pas nouvelle. Au milieu des années soixante-dix, Jean-Gustave Padioleau (1976) met en évidence une rhétorique journalistique ${ }^{8}$ dominante, celle de l'expertise critique. Elle s'incarne dans une forme textuelle en particulier, celle de l'exposé. Ce dernier, selon Padioleau, consiste en un "assemblage synthétique des dépêches d'agence, un rappel de données, l'introduction des connaissances tacites ou réflexives et des commentaires des rédacteurs spécialistes» (p. 92). Il consiste ainsi en un mélange de data et comporte une dimension normative. Mais il se distingue du journalisme d'opinion (tel qu'il s'exerce dans la presse de parti) car l'expression de jugements se fait sur la base d'une argumentation documentée et d'une compétence de spécialiste. Plus tard, Érik Neveu (1993) définit une autre forme textuelle que l'on peut aussi associer à la rhétorique de l'expertise critique, celle de l'analyse stratégique. Celle-ci se distingue de l'exposé par sa dimension de démontage ou de décryptage des stratégies politiques. Plus précisément, elle présente quatre caractéristiques principales. Elle utilise un vocabulaire concret, souvent imagé. Elle traduit les tactiques des acteurs politiques, leurs arrière-pensées, prend position sur leur habileté, leurs atouts, statue sur les conditions de succès de telle ou telle stratégie. Elle s'appuie sur la mémoire politique du journaliste, sa capacité à resituer les évènements dans le temps long, à les mettre en perspective, à éclairer le présent par le passé. Enfin, elle est mobilisée par des journalistes occupant des positions dominantes dans l'espace des rédactions (chefs de service, etc.). Au total, l'exposé et l'analyse stratégique proposent une lecture en surplomb de la vie politique, qui vise à une hauteur de vue sur les évènements politiques et à leur mise en perspective. La rhétorique de l'expertise critique correspond ainsi à une «stratégie du transfert de convic-

6. Le Monde, Libération, Le Figaro et Le Parisien.

7. De mi-novembre à mi-décembre 2001.

8. Le concept de rhétorique tel qu'il a été défini par Padioleau ne désigne pas seulement des procédures d'écriture, mais aussi des représentations et des pratiques journalistiques inscrites dans un rapport de force entre groupes de journalistes. Ces derniers s'approprient une certaine rhétorique journalistique qui leur sert de stratégie ou de ressource pour obtenir une reconnaissance de leur statut et de leur compétence. Par exemple, au milieu des années soixante-dix, les journalistes spécialistes de l'éducation au Monde se sont posés, face à leurs sources et à leurs collègues, auprès desquels ils avaient l'image de militants plutôt que de véritables journalistes, en experts critiques disposant d'une connaissance technique et approfondie des dossiers afin de gagner en légitimité. 
tion» (Lochard, 1996) quant à la façon dont il faut percevoir et appréhender le monde politique.

Nous faisons l'hypothèse qu'à partir des années quatre-vingt et surtout de la décennie quatre-vingt-dix, de nouvelles formes textuelles (le récit politique, les anecdotes politiques, le décryptage de la communication politique) mettent en scène une forme inédite de distance des journalistes politiques à leurs sources qui se distingue de la lecture en surplomb proposée par l'exposé ou l'analyse stratégique. Ces nouvelles formes textuelles, anecdotiques, familières, ancrées dans une forme quotidienne d'effet de réel, traduiraient un glissement progressif d'une rhétorique de l'expertise critique à une rhétorique du «cynisme» (Cappella, Jamieson, 1996; Schudson, 1999)9.

Nous proposons tout d'abord de décrire les formats d'expression préférentiels de la distance des journalistes à leurs sources à partir des années quatrevingt/quatre-vingt-dix (en nous concentrant sur l'exemple du récit politique), avant d'en analyser des principes de classification et d'en élaborer une cartographie, puis d'interroger les facteurs explicatifs de ce glissement d'une rhétorique à l'autre.

\section{De nouveaux formats d'expression de la distance des journalistes à leurs sources : l'exemple du récit politique}

À travers nos entretiens et nos observations directes, nous avons pu identifier trois formes textuelles régulièrement citées par les journalistes politiques et qui exprimeraient leur prise de distance à l'égard du personnel politique : le récit politique; le décryptage de la communication politique; les anecdotes politiques. Ces formes textuelles sont, sinon nouvelles, du moins jusque-là peu usitées dans la rubrique "Politique» des quotidiens nationaux. Certes, leur part dans la production quotidienne de l'information politique est certainement moindre par rapport à celle des évènements obligés, à l'instar des voyages présidentiels qui sont systématiquement couverts. Mais c'est moins la quantité de papiers écrits que leur statut d'article valorisant et valorisé aux yeux des journalistes qui nous intéresse ici. En effet, les journalistes politiques opèrent une hiérarchisation des formes textuelles - de la plus désuète ou inintéressante à la plus gratifiante - qui

9. Si le devoir d'irrespect et la dérision ne sont pas chose nouvelle dans la presse (Koren, 1996), c'est le fait que le cynisme s'étende à l'information politique dans les quotidiens nationaux qui nous intéresse ici, et plus encore, que cette posture devienne légitime et dominante dans les années quatre-vingt-dix. Pour Cappella et Jamieson (1996) et Schudson (1999), la notion de cynisme fait référence au strategic frame employé par les journalistes, qui représente la politique sous l'angle d'une lutte pour le pouvoir. Selon nous, d'autres dimensions du traitement journalistique participent de cette vision cynique de la politique, comme nous le verrons plus loin. 
traduit leur propre vision de l'excellence professionnelle ou - pour le dire autrement - leur rhétorique journalistique préférentielle.

Nous ne donnerons qu'une définition brève des formes textuelles de l'anecdote politique et du décryptage de la communication politique, pour nous pencher plus longuement sur celle du récit politique ${ }^{10}$. La première propose une lecture désacralisante ou désingularisante du champ politique qui donne à voir la vie politique par le petit bout de la lorgnette et les acteurs politiques comme des personnes parmi d'autres : à travers un format court, elle raconte des faits anecdotiques ou décalés, des à-côtés sous forme d'historiettes. La deuxième forme textuelle propose une lecture objectivante ou utilitariste de la vie politique qui donne à voir celle-ci comme un ensemble de stratégies pour l'accès au pouvoir et se concentre sur les affrontements personnels : il s'agit de révéler les coups médiatiques des élus et, par là, de signifier au lecteur que le journaliste n'est pas dupe des stratégies médiatiques des sources ${ }^{11}$.

Quant aux récits politiques, si les premiers sont publiés dès les années soixante-dix dans la presse commerciale et se diffusent dans la décennie quatre-vingt à l'initiative des quotidiens créés dans la décennie précédente, comme Le Quotidien de Paris, Le Matin de Paris ou encore Libération, ils ne s'imposent au Figaro et dans le journal de référence Le Monde que dans les années quatre-vingt-dix. Au départ, le récit politique souffrait en effet d'un déficit de légitimité : considéré comme un genre divertissant mais mineur, il était le fait de journalistes femmes, à l'instar de Françoise Berger à Libération, cantonnées à des spécialités dominées du journalisme politique ${ }^{12}$.

Le récit politique offre une lecture désacralisante de la vie politique. Les acteurs politiques sont à la fois présentés comme plus proches (des hommes et des femmes comme les autres) et montrés dans des situations malaisées, parfois ridicules.

Je me rappelle, par exemple, pendant la campagne de 95, on avait fait un papier sur la campagne des seconds couteaux, où on s'est amusé comme des petits fous parce qu'on suivait les partis des principaux candidats et puis, moi, on m'avait raconté des tonnes d'histoires là-dessus que j'avais trouvées formidables sur, effectivement, les seconds couteaux qui vont faire leur petite campagne et leurs petits meetings... Quand le grand chef réunit, je sais pas, 200 personnes, lui il en

10. La contrainte d'espace nous a conduit à ne développer que le cas du récit politique, qui nous semble le plus intéressant car il combine lecture désingularisante et lecture utilitariste de la vie politique.

11. Le décryptage de la communication politique se différencie de l'analyse stratégique en ce qu'il met en scène le média lui-même et les interactions entre les journalistes et le personnel politique. Voir Esser, Reinemann et Fan, 2001.

12. «Je me souviens qu'ici, très vite, [...] tout le monde riait des papiers de Françoise [Berger]. Les messieurs journalistes de la politique continuaient à trouver ça génial mais ne se sentaient pas menacés, oui, c'était un succès, mais dans leur esprit ça restait un genre mineur. » (Entretien, 14 octobre 2005) 
réunit 25 et puis quand l'autre est accueilli par les militants, dort dans un truc [à l'hôtel], etc., l'autre il prend son train de nuit et dans le train de nuit - c'était surtout ça l'image qui m'avait frappée parce que je sais qu'on l'avait raconté, c'était dans un corail entre Paris et Tulle, le dernier corail qui partait, qui rentrait à Paris genre à $1 \mathrm{~h}$ du matin, qui passait en gare de Tulle - s'étaient retrouvés avachis au bar qui était fermé ou dans la voiture, François Hollande à l'époque et je ne sais plus quels gens de droite qui rentraient chacun d'un meeting. C'était cette espèce de solidarité de la nuit des seconds couteaux ${ }^{13}$.

Le récit politique utilise un double registre d'empathie et d'ironie, de fascination et de répulsion.

Ça venait de la [...] démarche de parler plus des hommes qui faisaient la politique que des idées politiques qu'ils avaient. [...] On a beaucoup plus raconté les gens qui la faisaient et moi je sais que très franchement, ma passion, pourquoi j'ai aimé la politique, parce que j'ai aimé les gens qui la faisaient. Je trouve que c'est des drôles de bêtes, je trouve que c'est des gens qui sont intéressants même quand ils sont médiocres, et qu'à un moment donné ils ont choisi de s'exposer et ils sont plein de violence, ils se haïssent, ils se battent, ils souffrent parce qu'ils sont tout en haut et qu'ils redescendent tout en bas quand ils sont battus, parce que ce sont des hommes publics, comme des acteurs, je reviens à mon image de spectacle. Moi ce qui m'a profondément plu en politique, c'étaient ceux qui la faisaient. Voir de près un Chirac c'est formidable, voir de près un Jospin c'est formidable. Donc on est aussi plus attiré par ce qui les rend palpables, tout autant, voire plus que par leurs discours ou leur force politique proprement dite ${ }^{14}$.

En somme, le récit politique, d'une part, s'intéresse aux anecdotes, histoires, échos, bavardages, c'est-à-dire aux à-côtés de l'information politique obligée ou incontournable et, d'autre part, opère une personnalisation de la politique en portant attention aux personnages dans leurs traits de personnalité et de comportement, en les portraiturant.

Les explications suivantes pourront être lues à l'appui de l'exemple d'un récit politique publié dans Libération, que nous avons reproduit et commenté (figure 1, p. 120). Plusieurs procédés discursifs permettent d'exprimer cette lecture de la politique qui cherche à en montrer les coulisses comme si le lecteur y était. Les journalistes ont ainsi recours à ce que Roland Barthes (1982) a appelé «l'effet de réel », qui obéit principalementà trois procédés discursifs. Le premier est celui de la description (par opposition à la narration) ou de l'hypotypose (figure de style consistant à décrire une scène de manière si frappante qu'on croit la vivre), composée, selon l'auteur, de « détails inutiles», de «notations insignifiantes», et qui n'a d'autre fonction que de produire un « effet de réel» ou une «illusion référentielle»: elle dénote le «réel concret» (menus

13. Entretien, 22 mai 2003 : ancienne journaliste politique du Monde, diplômée de Sciences-Po Paris et du CFJ, âgée d'une quarantaine d'années.

14. Idem. 
gestes, attitudes transitoires, objets insignifiants, paroles redondantes), «l'avoir été là » des choses. Le deuxième réside dans la structure du récit sous la forme d'une succession temporelle d'actions, d'une transformation de certaines propriétés initiales des actants et d'une mise en intrigue (Adam, 1999; Charaudeau, Maingueneau, 2002, p. 239). Le troisième consiste à utiliser le discours rapporté (des énoncés qui conservent des indices de leur extériorité) ou le dialogue, en procédant à l'identification des sources (Charaudeau, 1997). L'effet de réel vise ainsi à produire un effet de preuve ou d'authentification de la scène rapportée par le journaliste. Il recherche aussi un effet de témoignage, en signalant par des embrayeurs ou des déictiques ${ }^{15}$ la présence du journaliste ou la présence de la situation d'énonciation. Ainsi, le journaliste est transformé en narrateur omniscient ou en témoin omniprésent (qu'il soit un témoin direct ou délégué).

Par ailleurs, le récit politique propose aussi une lecture utilitariste de la vie politique qui s'exprime notamment à travers un cadrage de la politique comme un jeu où s'affrontent des concurrents dont les choix stratégiques visent à la conquête du pouvoir. Les actions politiques sont analysées comme des coups tactiques, des logiques de carrières, des stratégies médiatiques.

Enfin, certains de ces procédés discursifs mettent en jeu la relation du journaliste à ses sources telle que ce premier veut la donner à voir. Ainsi, la sélection des éléments de discours à rapporter (partielle / totale), l’identification des locuteurs d'origine (partielle / totale / absente) et la manière de rapporter le discours d'origine (cité/intégré/narrativisé / évoqué) entrent dans des stratégies soit de mise à distance, soit, au contraire, d'appropriation (ou d'assumation) du discours de la source par le journaliste. La polyphonie (énoncés où plusieurs voix se font entendre simultanément) du récit politique correspond à plusieurs stratégies : rendre authentique en montrant qu'on rapporte les paroles mêmes; mettre à distance, parce que l'énonciateur n'adhère pas aux propos cités; se montrer objectif, sérieux. De même, la fonction expressive (Jakobson, 1963) est un autre procédé pour signaler le rapport que le journaliste entretient à ses sources. Par exemple, par l'ironie verbale ou par antiphrase, le journaliste subvertit la parole de la personne qu'il cible et produit ainsi un effet de mise à distance.

15. L'embrayage désigne l'ensemble des opérations par lesquelles un énoncé s'ancre dans sa situation d'énonciation et met donc en scène la présence du journaliste. Les embrayeurs (ou déictiques) de personnes («je», «mon», «le mien», etc.), temporels (les marques de présent/futur/passé attachées au verbe; les mots ou groupes de mots à valeur temporelle, comme "hier», «demain») et spatiaux (désignant le lieu, comme «ici», «là-bas», "ça»), constituent des marques linguistiques à travers lesquelles se manifeste l'énonciation. 
En somme, le récit politique propose une vision désacralisée et désacralisante de l'univers politique, ainsi qu'une lecture objectivante des règles du jeu et de la compétition politiques. Il correspond à une stratégie du dévoilement des coulisses de la politique et à une rhétorique du cynisme. Le terme cynisme désigne un mépris des conventions sociales, de l'opinion publique, des idées reçues, généralement fondé sur le refus de l'hypocrisie et/ou sur le désabusement, souvent avec une intention de provocation. Il signifie par extension affectation d'impudence, c'est-à-dire une attitude volontairement offensante, effrontée, ou contraire à la bienséance ${ }^{16}$. Certes, les journalistes dominants partagent avec le personnel politique certains principes de vision du monde (Neveu, 2003), mais leur posture d'écriture recouvre la dimension de désabusement et d'impudence comprise dans le cynisme.

Soulignons tout de même trois éléments de continuité entre le récit politique et les formes textuelles de l'exposé et de l'analyse stratégique : une définition institutionnelle de la politique et de ses frontières; une proximité obligée des journalistes à leurs sources (interdépendance fonctionnelle); une dimension microcosmique de l'information politique qui s'adresse avant tout à des lecteurs initiés.

Tableau 1:

Synthèse des caractéristiques du récit politique dans la presse quotidienne nationale

\begin{tabular}{|c|c|c|}
\hline & & Récit politique \\
\hline \multirow[t]{3}{*}{$\begin{array}{l}\text { Modes } \\
\text { de recueil des } \\
\text { informations }\end{array}$} & Journalistes & $\begin{array}{l}\text { - En majorité des femmes } \\
\text { - Couvrant des domaines de l'information } \\
\text { politique peu cotés }\end{array}$ \\
\hline & $\begin{array}{l}\text { Relation aux } \\
\text { sources }\end{array}$ & $\begin{array}{l}\text { - Proximité, entregent, familiarité } \\
\text { - Posture d'écoute, sens de la psychologie }\end{array}$ \\
\hline & Signature & - Collective (article écrit à plusieurs) \\
\hline \multirow{2}{*}{$\begin{array}{l}\text { Procédés } \\
\text { discursifs } \\
\text { et effets } \\
\text { recherchés }\end{array}$} & $\begin{array}{l}\text { «Effet de réel», } \\
\text { d'authentification } \\
\text { et de témoignage }\end{array}$ & $\begin{array}{l}\text { - Description } \\
\text { - Discours rapporté } \\
\text { - Embrayeurs }\end{array}$ \\
\hline & $\begin{array}{l}\text { Signaler } \\
\text { sa distance }\end{array}$ & $\begin{array}{l}\text { - Manières de rapporter le discours } \\
\text { - Ironie (fonction expressive ou émotive) } \\
\text { - Cadrage de la politique comme un jeu }\end{array}$ \\
\hline \multicolumn{2}{|c|}{ Lecture proposée } & $\begin{array}{l}\text { - Désacralisante ou « dé-singularisante» } \\
\text { - Objectivante ou utilitariste }\end{array}$ \\
\hline \multicolumn{2}{|l|}{ Rhétorique } & - Cynisme / stratégie du dévoilement \\
\hline
\end{tabular}

16. Définition issue du Trésor de la langue française. 
Figure 1 : Le récit politique à Libération. Une stratégie de dévoilement du monde politique

Quand Dany dîne chez son ami Lionel. " On se tutoie, c'est la tradition à gauche ", a décidé le chef du gouvernement.

Minuit sonne, et « Dany » est toujours là. Dans la salle à manger de « Lionel », qui lui a servi un steak, un bout de fromage, avec un peu de rouge. Ce qu'il y avait dans le frigo, en somme. C'était mardi soir, rue du Regard à Paris. Lionel Jospin recevait, chez lui, Daniel Cohn-Bendit. Sylviane, l'épouse, a salué l'invité, puis s'est éclipsée. Histoire de le (ou se) détendre, Jospin a dit, d'entrée : " On se tutoie, c'est la tradition à gauche ». La rencontre a failli devenir chimère, elle fut finalement des plus simples. Presque trop. Il y a une semaine, Matignon avait appelé le bureau bruxellois du député européen. Non sans avoir mûrement réfléchi au décor. La tête de liste des Verts aux européennes avait insisté pour être reçue par Jospin au lendemain du scrutin. Le recevoir très officiellement à Matignon, c'était reconnaître son score. Le rencontrer pour le sermonner, c'était risquer le coup de vieux. Jouer la complicité militante, le soir après le travail, c'est absorber un peu de l'effet CohnBendit. Jospin affable. Il était content, flatté, d'ailleurs, l'ancien leader de 68. Lui qui ne ratait jamais une pique contre ce Premier ministre qu'il n'avait jamais rencontré a eu droit au joli mois de mai de Jospin, alors fonctionnaire au Quai d'Orsay, droit de dire aussi au Premier ministre ce qu'il pense de la situation des sans-papiers ou encore des discours dominants sur la drogue. Et Lionel Jospin, étrangement affable, a dit : «Oui, ça, d'accord », puis : « Ça, je ne comprends pas ». Cohn-Bendit assure n'avoir pas même reçu un reproche pour ses attaques contre Jean-Pierre Chevènement. «On s'est expliqué nos positions en fonctions de notre histoire. Il m'a dit sa vision de la politique, m'a raconté ses ruptures.»
Les deux hommes ont même évoqués ces moments ou la politique « prend trop de place » dans la vie. Mais aucun n'a convaincu l'autre. Daniel Cohn-Bendit a répondu au Premier ministre qui l'interrogeait sur son envie de revenir en France, tout en assurant qu'il y avait peu de chances qu'il soit candidat à la mairie de Paris. Pour sa part, Lionel Jospin a fait comprendre au Vert, qui aime le chatouiller du côté de sa destinée présidentielle, qu'il ne tient pas à en parler: « Parce que je suis Premier ministre, parce que c'est contreproductif ». Retour à 0h30. Cohn-Bendit avait révisé avant de venir. Une heure avec Dominique Voynet, au ministère de l'environnement et de l'Aménagement du territoire. Rien ne s'est fait sans elle, qui avait même réitéré la demande de rendez-vous auprès du Premier ministre. Il avait aussi travaillé avec Pascal Perrineau, chercheur au Cevipof (centre d'étude de la vie politique), sur les enseignements des élections européennes. Il est donc venu chez Lionel Jospin avec quelques chiffres, pour lui expliquer « la perspective» des Verts, ou encore pourquoi il recommande pour les municipales un «premier tour identitaire » et « un manifeste de la gauche plurielle $»$ au second tour. Cohn-Bendit est parti à minuit trente, non sans avoir invité «Lionel » à venir le voir dans sa résidence secondaire du sud de la France. Hier matin, Matignon racontait un Jospin «très satisfait de sa soirée ». «Lionel » n'a même pas donné sa rituelle consigne de silence à son nouvel ami. Lequel était probablement invité à raconter en long et en large l'hospitalité de son hôte.

Copyright Libération, 10 septembre 1999, Judith Perrignon. 
Les procédés discursifs produisant un « effet de réel»:

- Le Discours Rapporté (souligné dans le texte) :

- de nombreux extraits (23 occurrences).

- La Description (hypotypose):

- déictiques temporels («minuit sonne», «mardi soir», « retour à oh30 », etc.) ; scène-type (" "Lionel”, qui lui a servi un steak un bout de fromage, avec un peu de rouge »);

- personnages et leur état émotionnel (« Jospin affable », « Il était content, flatté », etc.);

- mimétisme entre le registre de langue relâché du journaliste et la situation décrite comme détendue, «des plus simples » : «avec un peu de rouge » (métonymie).

- Le Témoignage :

- Le journaliste est un témoin « délégué » qui raconte à partir du témoignage de la source de l'information (vu et entendu par); celle-ci s'incarne dans le récit sous la forme d'un personnage (ici, Daniel Cohn-Bendit).

Les procédés discursifs produisant une lecture utilitariste du champ politique:

- Cadrage du jeu politique.

- Un élément d'analyse stratégique (style indirect libre en gras dans le texte).

- Signaler sa distance grâce à l'ironie (" son nouvel ami »).

- Manières de rapporter le discours :

- citations dominantes : " évoquées » (9 occurrences) et « directes» (6), qui marquent une distanciation du journaliste au dit rapporté ;

- citations secondaires : «narrativisées » (6) et « intégrées » (2), qui tendent à effacer la source et marquent l'appropriation («assumation ») du « dit rapporté » par le journaliste ;

- identification partielle des sources (en italique dans le texte), qui s'adresse à un lecteur initié, mais qui signale aussi une moindre déférence à l'égard du personnel politique : par le titre ou la fonction (12 fois); par le nom et le prénom (8); par le nom seul (7); par le seul prénom (7); par le prénom, le nom et la fonction (1). 
Plusieurs hypothèses, que nous ne développerons pas ici, peuvent être avancées pour expliquer l'usage du récit dans les pages Politique des quotidiens nationaux. Tout d'abord, il peut être lu comme un effet de la féminisation du journalisme politique. Ces innovations ont en effet été portées par des femmes occupant une position dominée dans les services politiques (Saitta, 2006). Ensuite, on peut supposer une convergence de ces nouveaux formats avec les attentes commerciales des directions de journaux : psychologisation, personnalisation (Neveu, 2000). Enfin, la plus grande accessibilité du personnel politique, le développement et la professionnalisation de la communication politique ont démultiplié les informations à disposition des journalistes. On observe, par ailleurs, des intérêts partagés entre les journalistes et le personnel politiques pour la promotion de récits à tendance biographique ou psychologisante (Le Bart, 2005), celle-ci s'inscrivant dans un processus de «privatisation» de la vie politique (Neveu, 2003).

\section{Cartographie des formats préférentiels d'expression de la distance des journalistes à leurs sources}

Après avoir décrit le fonctionnement des formats d'expression de la distance des journalistes politiques à leurs sources mobilisés depuis les années soixante-dix, nous proposons d'en élaborer une cartographie. À partir des propositions de différents auteurs pour catégoriser des genres journalistiques, nous avons construit trois axes.

Le premier, vertical, représente le «mode discursif dominant» (Charaudeau, 1997) de la forme textuelle. Il se décline en un pôle descriptif/narratif et un pôle argumentatif. Et se double d'une tension entre l'évènement rapporté (faits ou/et dits rapportés) et l'évènement commenté (le pourquoi et le comment de l'évènement rapporté).

Le deuxième axe, diagonal, représente l'ethos (au sens de Maingueneau, 2002). L'ethos désigne la façon dont l'énonciateur se laisse appréhender comme une voix et un corps, ou encore comme un caractère et une corporalité. Le deuxième axe se distribue entre un pôle «corporalisation » et un pôle «caractérisation» (Ringoot, Rochard, 2005). D'un côté, le corps et le faire du journaliste sont mis en scène dans l'article, le lecteur se trouve pris dans l'action avec le journaliste, de sorte que se crée entre eux une proximité physique et émotionnelle. De l'autre, le pensé et le ressenti du journaliste sont mis en scène, le lecteur est pris dans un dialogue virtuel avec le journaliste, de sorte que se crée entre eux une proximité intellectuelle et émotionnelle.

Le troisième axe représente la «visée communicative» (Lochard, 1996) de la forme textuelle. Il est composé de deux pôles représentant, d'un côté, une visée informative et, de l'autre, une visée persuasive. Ou encore, on pourrait 
reprendre là la distinction faite par Ruth Amossy (Amossy, Koren, 2004) entre la dimension et la visée argumentatives. La première désigne un discours qui n'a pas l'intention délibérée d'agir sur l'auditoire en lui faisant adopter une position déterminée mais qui n'en oriente pas moins sa réflexion, tandis que la seconde indique une prise de parole qui entend défendre une thèse et agir surl'auditoire.

Les formes textuelles situées dans la partie supérieure gauche de la figure 2 correspondent à une rhétorique du « cynisme » visant au dévoilement des coulisses de la politique : le journaliste se pose comme témoin, il crée un «effet de réel » visant à faire croire à la réalité de ce qu'il dit et opère ainsi une « transformation réaliste» (Barthes, 1982).

Les formes textuelles situées dans la partie inférieure droite correspondent à une rhétorique de l'expertise critique visant au «transfert de conviction sur la façon dont il faut percevoir et appréhender » (Lochard, 1996) le monde politique : par des jeux démonstratifs et une posture évaluative, prescriptive, le journaliste met en scène son pensé; il adopte une stratégie didactique.

Figure 2 : Cartographie des formats d'expression de la distance des journalistes politiques à leurs sources

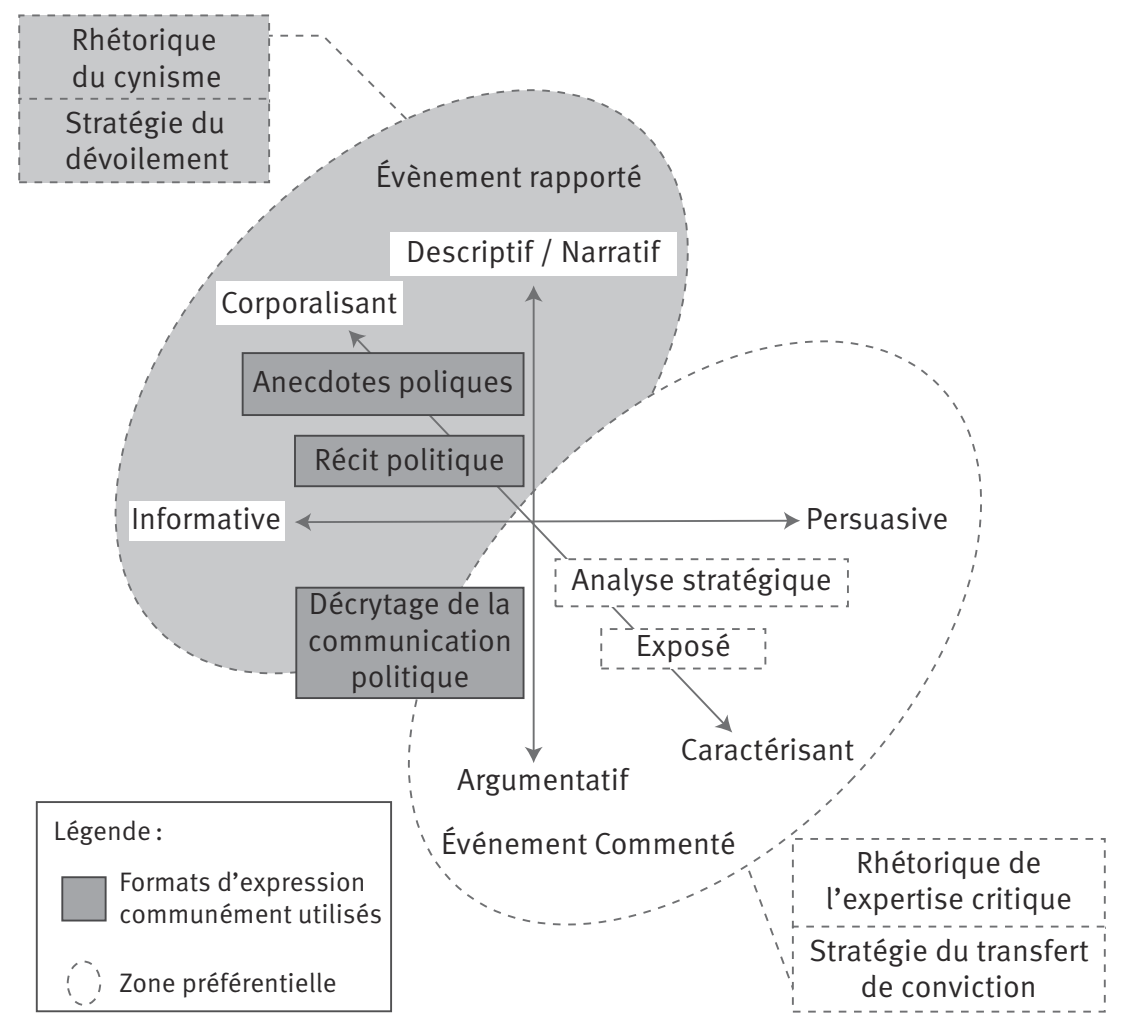




\section{EugÉNIE SAITTA}

Globalement, les journalistes politiques délaissent dans une certaine mesure la rhétorique de l'expertise critique pour investir plus fortement la rhétorique du «cynisme». Dans la figure 2, l'espace des formats d'expression de la distance qu'ils mobilisent de façon préférentielle correspond à la moitié gauche. Ce glissement d'une rhétorique à l'autre s'effectue au cours des années quatre-vingt/quatre-vingt-dix. Il révèle une évolution dans le type de mise à distance que les journalistes politiques opèrent à l'égard de leurs sources.

\section{Les facteurs explicatifs d'un glissement de la rhétorique de l'expertise critique à une rhétorique du «cynisme " au cours des années quatre-vingt/quatre-vingt-dix}

Le glissement d'une rhétorique de l'expertise critique à une rhétorique du «cynisme» s'explique à la fois par l'évolution des interdépendances entre journalisme et politique et par les transformations du champ journalistique depuis les années quatre-vingt/quatre-vingt-dix ${ }^{17}$ (tableau 2, p. 126).

Premier élément d'explication : ce glissement correspond à un infléchissement de l' «enchantement» politique des journalistes politiques. Alors que la rhétorique de l'expertise critique impliquait une adhésion aux principes de fonctionnement du champ politique, un enchantement, la rhétorique du "cynisme» provient d'un désenchantement qui prend la forme d'une "critique politisée» (Gaxie, 2001) ${ }^{18}$. Ainsi, la croyance des journalistes politiques en la capacité de la politique à changer le monde, leur investissement dans un clivage droite/gauche perçu comme porteur de sens et de projets politiques et leur respect pour la classe politique sont amoindris. Cette évolution est à mettre en lien avec les transformations des modes de socialisation politique et professionnelle des journalistes politiques, de leur parcours de formation et de leurs repères cognitifs, de leurs caractéristiques sociographiques (féminisation). En somme, on voit là les effets de l'arrivée d'une nouvelle génération de journalistes politiques à partir de la fin des années quatre-vingt et, surtout, des transformations dans le recrutement et la gestion des ressources humaines dans les quotidiens nationaux.

Deuxième élément d'explication : à partir des années quatre-vingt, le désengagement partisan des quotidiens nationaux qui se désolidarisent des positionnements de partis qu'ils avaient soutenus jusque-là va de pair avec une injonction à l'objectivité journalistique. L'élection de François Mitterrand à la présidence de la République en 1981 a contribué à une neutralisation des

17. Pour de plus amples développements, voir Saitta, 2006.

18. Toutefois, ce désenchantement ne signifie pas que l'illusio politique ne fonctionne plus chez les journalistes politiques. Au contraire, ces derniers adhèrent aux règles du jeu politique et $y$ restent fortement investis. 
journaux de gauche qui s'est traduite, par exemple à Libération, cette année-là, par la mise à l'écart des journalistes réputés les plus militants (juhem, 2001). Cette objectivité est avant tout formelle. Elle s'exprime par exemple dans la séparation physique des faits et des commentaires, ces derniers étant circonscrits dans des espaces à part, comme les rubriques «Débats et opinions» au Figaro, "Rebonds» à Libération à partir de 1988, "Horizons-Débats» au Monde à partir de 1989 ou encore "Tribune libre» à L'Humanité à partir de 1999. Ces espaces sont réservés à des paroles extérieures, ce qui permet aux journaux de se désolidariser des opinions qui s'expriment dans leurs pages (Lettieri, 2002). De même, l'éditorial qui, par son genre, est pourtant autorisé à pratiquer l'engagement personnel, connait un processus «d'objectivation discursive». Koren (2004) met en évidence l'usage d'une «rhétorique de la neutralisation de la prise de position » dans l'éditorial et Jufer et Herman (2001) montrent comment, en parlant de plus en plus au nom d'une opinion publique, la voix de l'éditorialiste tend à se désubjectiviser. Ainsi le droit à la parole des journalistes politiques est-il conditionné par le recours aux apparences neutres ou aux effets d'objectivité.

Troisième élément d'explication : les contraintes économiques et professionnelles se conjuguent pour délégitimer le modèle d'excellence professionnelle porté par le journalisme politique. D'un côté, on constate une inadaptation de la rhétorique de l'expertise critique et de ses formes textuelles préférentielles aux impératifs commerciaux : par exemple, la réduction de la taille moyenne des articles décourage les longs développements qu'on trouvait dans l'exposé ou l'analyse stratégique. D'un autre côté, on constate une stigmatisation de la rhétorique de l'expertise critique qui ne correspond pas aux standards professionnels prônés par un groupe concurrent et montant au sein des rédactions : les journalistes d'investigation et les reporters issus des Informations générales. La rhétorique de l'expertise critique se voit critiquée pour sa dimension littéraire et normative, son ésotérisme et les rapports de proximité avec le personnel politique, qu'elle rend nécessaires de la part des journalistes.

En somme, ce passage tendanciel d'une rhétorique à une autre peut se lire comme l'effet d'un triple processus de dépolitisation du journalisme politique, entendue comme un désenchantement politique des journalistes, un désengagement partisan des titres de presse et enfin un déclassement du journalisme politique au sein des rédactions de la presse quotidienne nationale. 
Tableau 2 : Les facteurs explicatifs d'un glissement d'une rhétorique de l'expertise critique à une rhétorique du «cynisme " au tournant des années quatre-vingt-dix

\begin{tabular}{|c|c|c|c|}
\hline & & $\begin{array}{c}\text { Rhétorique de } \\
\text { l'expertise critique }\end{array}$ & $\begin{array}{l}\text { Rhétorique du } \\
\text { «cynisme» }\end{array}$ \\
\hline \multicolumn{2}{|l|}{ Période } & $\begin{array}{l}\text { Années soixante- } \\
\text { dix/ début des } \\
\text { années quatre-vingt }\end{array}$ & $\begin{array}{l}\text { À partir des années } \\
\text { quatre-vingt / quatre- } \\
\text { vingt-dix }\end{array}$ \\
\hline \multirow{2}{*}{$\begin{array}{l}\text { État des } \\
\text { relations } \\
\text { entre } \\
\text { champs } \\
\text { journalis- } \\
\text { tique et } \\
\text { politique }\end{array}$} & $\begin{array}{l}\text { Structuration } \\
\text { de la presse } \\
\text { quotidienne } \\
\text { nationale }\end{array}$ & $\begin{array}{l}\text { - Presse } \\
\text { polarisée (majo- } \\
\text { rité / opposition; } \\
\text { droite / gauche) }\end{array}$ & $\begin{array}{l}\text { - Presse désengagée } \\
\text { des positionnements } \\
\text { partisans / Logique } \\
\text { commerciale } \\
\text { dominante }\end{array}$ \\
\hline & $\begin{array}{l}\text { Rapport des } \\
\text { journalistes au } \\
\text { politique }\end{array}$ & $\begin{array}{l}\text { - Engagement poli- } \\
\text { tique et militant } \\
\text { - «Enchantement» }\end{array}$ & $\begin{array}{l}\text { - Désengagement } \\
\text { - «Désenchantement» } \\
\text { et «critique politisée» }\end{array}$ \\
\hline \multirow{2}{*}{$\begin{array}{l}\text { Transforma- } \\
\text { tions } \\
\text { du champ } \\
\text { journalis- } \\
\text { tique }\end{array}$} & $\begin{array}{l}\text { Impératifs } \\
\text { économiques }\end{array}$ & $\begin{array}{l}\text { - Pression } \\
\text { économique } \\
\text { moindre }\end{array}$ & $\begin{array}{l}\text { - Hyperconcurrence } \\
\text { du marché } \\
\text { journalistique }\end{array}$ \\
\hline & $\begin{array}{l}\text { Réorganisa- } \\
\text { tion des hiérar- } \\
\text { chies profes- } \\
\text { sionnelles }\end{array}$ & $\begin{array}{l}\text { - Position } \\
\text { dominante des } \\
\text { journalistes } \\
\text { politiques au sein } \\
\text { des rédactions }\end{array}$ & $\begin{array}{l}\text { - Déclassement } \\
\text { du journalisme } \\
\text { politique }\end{array}$ \\
\hline \multicolumn{2}{|c|}{$\begin{array}{l}\text { Formats d'expression } \\
\text { préférentiels }\end{array}$} & $\begin{array}{l}\text { - Exposé } \\
\text { - Analyse } \\
\text { stratégique } \\
\text { - Éditorial }\end{array}$ & $\begin{array}{l}\text { - Récit politique } \\
\text { - Décryptage de la com- } \\
\text { munication politique } \\
\text { - Anecdotes et } \\
\text { «à-côtés» } \\
\text { politiques }\end{array}$ \\
\hline \multicolumn{2}{|c|}{$\begin{array}{l}\text { Formes de distanciation } \\
\text { aux sources }\end{array}$} & $\begin{array}{l}\text { - Lecture en } \\
\text { surplomb de la } \\
\text { politique (prise de } \\
\text { hauteur et mise en } \\
\text { perspective) }\end{array}$ & $\begin{array}{l}\text { - Lecture utilitariste de } \\
\text { la politique } \\
\text { - Lecture désacralisante } \\
\text { de la } \\
\text { politique }\end{array}$ \\
\hline \multicolumn{2}{|c|}{$\begin{array}{l}\text { Stratégie } \\
\text { de communication }\end{array}$} & $\begin{array}{l}\text { - Transfert de } \\
\text { conviction sur la } \\
\text { façon dont il faut } \\
\text { percevoir et } \\
\text { appréhenderle } \\
\text { monde politique }\end{array}$ & $\begin{array}{l}\text { - Dévoilement } \\
\text { des coulisses de } \\
\text { la politique }\end{array}$ \\
\hline
\end{tabular}




\section{Références}

AdAM Jean-Michel, 1999, Le récit, Paris, PUF.

Amossy Ruth, Koren Roselyne, 2004, "Présentation», Semen, n 17, http://semen. revues.org/document2305.html

BARTHES Roland, 1982, "L'effet de réel», R. Barthes, G. Genette, T. Todorov éd., Littérature et réalité, Paris, Le Seuil, p. 81-90.

CAPpella Joseph N., Jamieson Kathleen H., 1996, «News frames, political cynicism and media cynicism », The Annals of the American Academy of Political and Social Science, vol. 546, $\mathrm{n}^{\circ}$ 1, p. 71-84.

Charaudeau Patrick, 1997, Le discours de l'information médiatique. La construction du miroir social, Paris, Nathan.

Charaudeau Patrick, Maingueneau Dominique, 2002, Dictionnaire d'analyse et de discours, Paris, Le Seuil.

ESSER Franck etal., 2001, "Spin doctors in the United States, Great Britain and Germany. Metacommunication about media manipulation », Press/Politics, vol. 6, p. 16-45.

GAXIE Daniel, 2001, «Les critiques profanes de la politique. Enchantements, désenchantements, réenchantements», J.-L. Briquet, P. Garraud éd., Juger la politique, Rennes, Presses universitaires de Rennes, p. 217-240.

HeRmAn Thierry, Jufer Nicole, 2001, "L'éditorial, "vitrine idéologique du journal” ?", Semen, $\mathrm{n}^{\circ} 13$.

JAковSON Roman, 1963, Essais de linguistique générale. T. 1: Lesfondations du langage, Paris, Minuit.

JUHEM Philippe, 2001, «Alternances politiques et transformation du champ de l'information en France après $1981 »$, Politix, nº 56, p. 185-208.

KoRen Roselyne, 2004, "Argumentation, enjeux et pratique de l'“engagement neutre" : le cas de l'écriture de presse», Semen, ${ }^{\circ}$ 17, http://semen.revues.org/ document2308.html

- 1996, Les enjeux éthiques de l'écriture de presse et la mise en mots du terrorisme, Paris, Montréal, L'Harmattan.

LE BART Christian, 2005, «La construction sociale du genre “livre politique” », L. Arnaud, C. Guionnet éd., Les frontières du politique, Rennes, Presses universitaires de Rennes, p. 27-48.

LetTIERI Carmela, 2002, «Formes et acteurs des débats publics contemporains. Les tribunes publiées dans la presse quotidienne en Italie et en France», doctorat de sciences de l'information et de la communication sous la direction de Rémy Rieffel, IFP, Université Paris 2.

LOCHARD Guy, 1996, "Genres rédactionnels et appréhension de l'événement médiatique. Vers un déclin des modes “configurants" ?", Réseaux, n 76, p. 3-102.

Neveu Érik, 2003, "Privatisation et informalisation de la vie politique», Y. Bonny, J.-M. de Queiroz, É. Neveu éd., Norbert Elias et la théorie de la civilisation. Lectures et critiques, Rennes, Presses universitaires de Rennes, p. 185-207. 
- 2000, «Le genre du journalisme. Des ambivalences de la féminisation d'une profession », Politix, vol. 13, n ${ }^{\circ}$ 1, p. 179-212.

- 1993, "Pages “Politique” », Mots. Les langages du politique, n 37, p. 6-28.

PADIOLEAUJean-Gustave, 1976, "Systèmes d'interactionetrhétoriquesjournalistiques », Sociologie du travail, vol. $18, \mathrm{n}^{\circ}$ 3, p. 256-282.

RINGOOT Roselyne, RochaRd Yves, 2005, «Proximité éditoriale : normes et usages des genres journalistiques», Mots. Les langages du politique, $\mathrm{n}^{\circ}$ 77, p. 73-91.

SAITTA Eugénie, 2006, "Les transformations du journalisme politique depuis les années quatre-vingt. Une comparaison France / Italie», doctorat de science politique sous la direction d’Érik Neveu, Université Rennes 1.

SCHUDSON Michael, 1999, "Social origins of press cynism in portraying politics», American Behaviorist Scientist, vol. 42, nº 6, p. 998-1008. 